\title{
L'affaire Romand, du fait divers au cinéma. Penser les circulations stylistiques et discursives d'un genre culturel dévalué
}

The Romand Case, from fait divers to Film. The Stylistic and Discursive

Circulations of a Devalued Cultural Genre

\section{Sophie Dubec}

\section{OpenEdition \\ Journals}

Édition électronique

URL : http://journals.openedition.org/recherchestravaux/973

ISSN : 1969-6434

Éditeur

UGA Éditions/Université Grenoble Alpes

Édition imprimée

ISBN : 978-2-37747-056-3

ISSN : 0151-1874

Référence électronique

Sophie Dubec, «L'affaire Romand, du fait divers au cinéma. Penser les circulations stylistiques et discursives d'un genre culturel dévalué », Recherches \& Travaux [En ligne], 92 | 2018, mis en ligne le 01 juin 2018, consulté le 08 septembre 2020. URL : http://journals.openedition.org/recherchestravaux/ 973

Ce document a été généré automatiquement le 8 septembre 2020

(C) Recherches \& Travaux 


\title{
L'affaire Romand, du fait divers au cinéma. Penser les circulations stylistiques et discursives d'un genre culturel dévalué
}

\author{
The Romand Case, from fait divers to Film. The Stylistic and Discursive \\ Circulations of a Devalued Cultural Genre
}

Sophie Dubec

1 Le 9 janvier 1991, Jean-Claude Romand tuait sa femme, ses enfants, ses parents et son chien avant de tenter de se suicider, alors que sa double vie était sur le point d'être découverte. Faux médecin, chercheur fictif à l'OMS, il avait menti 18 ans durant à son entourage, vivant de l'argent qu'il leur soutirait sous prétexte d'intéressants placements financiers. Mythomane professionnel, escroc consciencieux, familicide... le meurtrier défraya durablement la chronique et fit l'objet de nombreuses reprises médiatiques et artistiques. Cette étude se propose d'appréhender deux d'entre elles, des productions cinématographiques : L'Emploi du temps de Laurent Cantet et L'Adversaire de Nicole Garcia.

2 Comment analyser ces phénomènes de circulation ? Produites à l'orée des années 2000, les deux fictions entretiennent des rapports différenciés à l'affaire : adapté de l'ouvrage éponyme d'Emmanuel Carrère, L'Adversaire (2002) restitue de façon relativement fidèle l'histoire du faux médecin, rebaptisé Jean-Marc Faure dans le film ; L'Emploi du temps (2001) s'en détache quant à lui sensiblement : il met en scène la vie d'un cadre licencié, Vincent, s'inventant durant quelques mois un emploi dans une filiale de l'OMS avant de revenir, une fois démasqué, dans le droit chemin du travail. Matériau narratif puissant, l'affaire Romand doit également être appréhendée du point de vue de sa dimension fait-diversière. Or, les relations entre faits divers et arts, si elles ne sont pas nouvelles, sont loin d'être indemnes d'ambiguïtés : loués pour les cristallisations qu'ils opèrent de 
problématiques tant humaines que sociales, les faits divers sont fréquemment dévalués dans leur matérialité, soit leur médiation journalistique ${ }^{1}$.

Cette situation paradoxale, d'attirance et de rejet, invite à repenser la notion de genre qui, loin de ne renvoyer qu'à des considérations formelles, sera ici conçue comme catégorie culturelle. Il s'agira en d'autres termes de dépasser l'illusion naturaliste produite par les catégorisations pour considérer le genre comme une pratique discursive, située à la croisée de nombreux rapports de pouvoir. Ce détour théorique nous permettra de nous émanciper du registre de la sublimation, souvent mobilisé pour caractériser le passage des médias aux arts, et de reconsidérer tant les appropriations que les points de convergence entre faits divers et fictions cinématographiques. Notre analyse s'effectuera ensuite en deux temps. Nous montrerons tout d'abord combien les appropriations et jeux stylistiques engagés avec le fait divers dépendent des façons dont le genre culturel est problématisé au sein des deux productions, récusant ainsi toute réification de cette matière indisciplinée. Nous appréhenderons dans un second temps le fait divers comme discours, historiquement et socialement situé, dans le but de réfléchir au formidable attrait que suscite ce matériau journalistique. Nous faisons ainsi l'hypothèse que, si ces récits criminels inspirent tant, c'est que les façons dont ils mettent en scène les désordres quotidiens sont socioculturellement signifiantes.

\section{Le fait divers comme genre culturel}

Le fait divers se présente à la fois comme une provocation et une invitation à repenser le concept de genre. Transmédiatique et protéiforme, il souligne la faiblesse du paradigme textualiste qui, centré sur l'identification de propriétés formelles, ne parvient pas à circonscrire l'hétérogénéité de ses manifestations culturelles. Objet de définitions contradictoires entre professionnels et chercheurs, s'incarnant dans des types de discours aussi variés que la brève journalistique ou la chronique judiciaire, le fait divers semble échapper à toute entreprise de définition. C'est pourquoi Annik Dubied et Marc Lits préconisent de l'appréhender par le biais de la théorie des prototypes: «Il faudra admettre que le fait divers est une catégorie aux frontières flottantes, dans laquelle les éléments gravitent plus ou moins près d'un épicentre ${ }^{2}$.» Le catalogue hétérogène auquel ils aboutissent contraste cependant avec la connaissance intuitive à laquelle fait appel ce genre ${ }^{3}$. Cet écart entre, d'une part, la résistance du fait divers à toute entreprise de définition stricte et, d'autre part, la conception relativement précise qu'en ont les acteurs, invite ainsi à un déplacement du regard, qui considérerait le fait divers comme catégorie culturelle. Il s'agit, dans cette perspective, de ne plus penser le genre du point de vue de ses propriétés internes, mais d'aller audelà du texte pour envisager certaines des interactions qui lui donnent naissance. Telle est la proposition de Jason Mittell qui, préconisant de considérer le genre comme pratique discursive, s'interroge non plus sur "ce que signifie un genre particulier " mais davantage "sur les façons dont il est défini collectivement ${ }^{4}$ ». Une telle approche permet dès lors de dépasser l'illusion naturaliste produite par les catégorisations pour s'intéresser à ce qui, précisément, génère ces catégorisations. Historicisés et complexifiés, les genres s'apparentent aux formations discursives foucaldiennes, soit « des systèmes de pensées historiquement spécifiques, des catégories conceptuelles qui concourent à définir des expériences culturelles au sein d'un système de pouvoir plus large ${ }^{5} »$. 
5 De ce point de vue, le fait divers n'est pas avant toute chose « une mise en scène sémiotique ${ }^{6}$ » mais devient le produit de discours et pratiques multiples qui, en s'interconnectant, concourent à lui donner sa signification, ses matérialisations autant que sa valeur sociale. Le primat des rapports de pouvoir sur les mises en scène langagières et/ou visuelles permet de considérer non plus seulement les discours participant au modelage des faits divers, mais également ceux participant de leur perception. Or, le fait divers comme genre culturel semble souffrir au moins d'une double dévaluation. Au sein des discours d'information, il est opposé à des productions jugées plus sérieuses, à l'image des informations internationales ou traitant de «la » politique. Cette dichotomie semble susceptible d'expliquer les résistances toujours prégnantes pour conférer aux faits divers une dimension collective: discours de dérogation aux normes, ils restent paradoxalement traités par certains travaux comme des informations apolitiques, ayant peu de portée sociale ${ }^{7}$. Àcette première hiérarchisation se superpose une deuxième, entre culture populaire et culture légitime : identifiés comme des productions de masse, les faits divers demeurent encore pensés comme des informations trompeuses voire trompées qui, dictées par la recherche d'une audience maximale, joueraient sur les «bas instincts» de publics forcément crédules. Simpliste, cette bipartition n'en reste pas moins socialement structurante et se décline en plusieurs versions. Elle participe à appréhender la circulation des faits divers dans les arts du point de vue de la sublimation. Ce registre est perceptible dans la démarche portée par certains artistes : "Visiblement conscients des critiques que suscite leur matière première (manque d'imagination, voyeurisme, pillage, irrespect), les auteurs insistent généralement sur le fait qu'ils confèrent un sens à une réalité qui en manque cruellement ${ }^{8}$. " Mais il imprègne également tout un pan de la littérature académique situant, à la suite des travaux de Roland Barthes', le fait divers journalistique du côté de l'insignifiant ou encore reproduisant la condamnation bien connue de la spectacularisation de l'information ${ }^{10}$.

6 L'approche culturelle des faits divers permet dès lors de se départir d'un double écueil méthodologique: celui de reproduire, tout d'abord, une grille de lecture élitiste, porteuse d'une violence symbolique non négligeable ; celui, ensuite, de considérer que les reproches adressés aux faits divers correspondent effectivement à leur "réalité ". La mise à distance de ces discours critiques n'implique nullement de verser dans le relativisme; il ne s'agit pas, en d'autres termes, d'avancer que "tout se vaut». Contre le registre de la sublimation nous avançons cependant que la prise en compte de la spécificité de ces objets implique de les distinguer radicalement, et de renoncer à tout jugement en termes de hiérarchie culturelle.

\section{Romand à l'écran : (en)jeux du fait divers}

7 Objet complexe, le fait divers ne peut être réduit à sa dimension proprement matérielle. C'est pourquoi une analyse des appropriations de ce genre médiatique doit s'efforcer de rendre compte des problématisations qu'en effectuent les œuvres pour ensuite envisager les rapports qu'elles entretiennent avec cette matière culturelle, notamment sur le plan stylistique. Étudier la circulation du fait divers n'implique pas, dans cette perspective, un retour au paradigme textualiste, le langage fait-diversier prenant toujours sens vis-à-vis des représentations circulant à son égard. 


\section{Fait divers médiatique ou affaire criminelle?}

8 Si les rencontres entre faits divers et productions artistiques sont souvent d'ordre conflictuel ${ }^{11}$, rien de tel ne transparaît dans les deux films inspirés de l'affaire Romand. L'Emploi du temps et L'Adversaire ont en commun de ne ménager aucune place à la dimension journalistique : rien n'est dit, ou rapporté, des professionnels et de leur mise en discours dans ces deux productions. Cette évacuation mène dès lors à se questionner sur l'objet même de l'appropriation: le cas Romand imprègne-t-il les deux longsmétrages en tant que fait divers journalistique ou comme affaire criminelle relativement autonome? Cette incertitude transparaît en effet dans les filiations que les cinéastes attribuent à leur film. Rebutée par l'horreur du drame au moment de la médiatisation, Nicole Garcia revendique ainsi un intermédiaire littéraire : "c'est le livre d'Emmanuel Carrère qui m'a ouvert une brèche dans l'opacité du fait divers. [...] Il m'a permis d'identifier, au creux de cet incroyable cauchemar, une histoire simple ${ }^{12}$.» Et elle gomme par la suite toutes les références journalistiques présentes dans l'ouvrage d'origine. Cette distanciation paraît encore plus radicale chez Laurent Cantet, qui affirme que ni le fait divers, ni l'affaire criminelle, n'ont motivé sa démarche. Parti du projet de « raconter l'histoire d'un individu cherchant à échapper aux contraintes du boulot ", le réalisateur et son co-scénariste ont fait le lien avec le meurtrier dans un second temps : «Un fait divers, c'est quelque chose de tellement riche dans le condensé qu'il offre de la dimension humaine, de possibilités de suspense. [...] en même temps, l'envie de fiction dépassait la restitution du fait ${ }^{13}$. "S'inspirant librement de la vie de Jean-Claude Romand, dont il reprend la fiction de travail et les malversations financières, L'Emploi du temps effectue de nombreuses coupes dans l'histoire originelle, allant jusqu'à vider le fait divers de sa substance: les meurtres et la dimension pathologique du personnage principal sont ainsi absents du scénario.

9 La médiation fait-diversière, si elle ne fait pas l'objet de critiques directes dans les deux fictions, n'en demeure pas moins radicalement mise à distance, comme effacée par la célébrité du faux médecin. Ce rapport ambigu s'accentue lorsqu'on observe les moyens par lesquels Nicole Garcia et Laurent Cantet cherchent à s'émanciper de cet hypotexte collant. Divers procédés semblent être mis en place pour contrebalancer l'empreinte durable du fait divers dans les mémoires collectives du crime.

On trouve ainsi dans L'Adversaire une véritable revendication fictionnelle. Ce positionnement est affiché dès l'ouverture du film par la hiérarchisation de trois épigraphes : le premier ancre l'univers diégétique du film dans la fiction, en changeant le nom du personnage principal («Pendant quinze ans, tout le monde a cru Jean-Marc Faure. Sa femme, ses parents, ses amis »), le deuxième donne un indice de sa filiation littéraire avec une citation non explicitée d'Emmanuel Carrère («Il y a pire qu'être démasqué, c'est de ne pas être démasqué. »), le troisième enfin établit un lien discret à l'affaire Romand, par le biais de la caution du réel («Ce film est inspiré d'une histoire vraie. »). L'Adversaire multiplie ensuite les signes d'appartenance fictionnelle : le choix d'une star française - Daniel Auteuil - pour incarner le rôle principal contrebalance la célébrité du faux docteur; la musique de Badalamenti ou encore la reprise de codes esthétiques $\mathrm{du}$ film noir installent quant à eux L'Adversaire dans une stylistique cinématographique. Ces différents jeux intertextuels confèrent une dimension métadiscursive à la mise en scène, signifiant une appropriation particulière du drame. 
11 Dans la mesure où il n'établit pas de filiation directe avec Romand, L'Emploi du temps entretient une tout autre relation avec le fait divers : radicalement mise à distance, l'affaire hante paradoxalement l'ensemble du film, la pré-connaissance de l'histoire créant un effet de suspense. Ce jeu sur les attentes permet de souligner le drame réel, le suicide symbolique du protagoniste lorsque, succombant aux attentes paternelles et familiales, il se rend dans la dernière séquence à un entretien d'embauche. Cette thèse est notamment évoquée par Yannick Lebtahi et Isabelle Roussel-Gillet : « Vincent fugue par la fenêtre et ne se défenestre pas, Vincent quitte la voiture au bord de l'autoroute pour encore marquer sa fuite et sa distance. Cette avant-dernière séquence a pu être interprétée comme un suicide avorté (Lefort), une fin déjouée par le long entretien final $^{14}$. »

\section{Les (non-)styles du fait divers : ennoblissement de la trivialité et renversement de scénario}

12 Fait divers, affaire criminelle : l'autonomisation de l'affaire Romand comme trame narrative n'élimine pas pour autant l'empreinte journalistique bien présente dans les mémoires du drame. La transformation d'un crime individuel en fait divers puis en « affaire » implique en effet l'inscription dans les imaginaires de traces laissées par la médiation fait-diversière. Chacune à leur façon, les fictions semblent ainsi cristalliser des problématisations distinctes du genre culturel, donnant lieu à des jeux et contrastes stylistiques différenciés.

13 À la suite de Carrère, Nicole Garcia insuffle une dimension tragique au fait divers originel, qu'elle dit vouloir émanciper de son aspect trivial ${ }^{15}$. Cette transposition s'exprime d'un point de vue stylistique par une esthétique dramatique, aux tonalités théâtrales, participant d'une forme d'ennoblissement du fait divers. Ce parti pris est surtout perceptible dans les scènes retraçant l'errance de Jean-Marc Faure dans le salon familial la journée suivant les meurtres. La mise en scène saturée relève ici d'une forme de classicisme : la lumière fortement contrastée, rappelant l'expressionnisme allemand, enferme le personnage dans l'obscurité, soulignant sa part d'ombre; les nombreuses traces du meurtres - tache sur l'épaule, bol de céréales brisé sur le sol, arbre tombé dans le jardin - confèrent au lieu et aux objets une symbolique marquée ; les flashbacks subjectifs du personnage vers des scènes de famille joyeuses soulignent son emprisonnement mental. Cette relecture esthétique du fait divers s'accompagne, sur le plan narratif, d'un déplacement de l'écriture fait-diversière. Nicole Garcia revisite les pratiques d'enquête généralement observées dans les récits criminels. Reprenant la structure de l'entonnoir inversé, le film débute une fois les meurtres accomplis, et procède ensuite par succession de flashbacks et de flashforwards pour tenter de restituer l'intelligibilité du drame. La narration filmique prend ainsi le caractère fragmenté des récits journalistiques, notamment par la multiplication des instances énonciatrices (interrogatoires des proches, aveux enregistrés de Jean-Marc Faure). Pourtant, l'enquête proposée par L'Adversaire a ceci de particulier qu'elle ne cherche nullement à multiplier les discours donnant accès à l'intériorité du meurtrier ou conférant une causalité à ses actes ; la caméra omnisciente navigue au contraire de scènes en récits pour montrer comment les mensonges du personnage se resserrent progressivement autour de lui : il s'agit en d'autres termes de restituer la logique du meurtre, non son fondement. 
14 Le film de Laurent Cantet laisse entrevoir une tout autre appréhension du fait divers, conçu ici comme scénario macabre et exceptionnel. Le renversement de ce script apparaît central dans la construction du film, mettant en lumière sa dimension politique : l'effacement de la trame meurtrière permet en effet de souligner la vraie tragédie du personnage, celle de sa soumission finale aux injonctions identitaires de la famille bourgeoise et du monde du travail. Ce contraste semble également signifié par des partis pris dédramatisants, opposant à la radicalité attribuée au fait divers un monde étrangement normal et profondément normatif. La mise en scène réaliste des scènes familiales et des lieux de travail contraste ainsi avec des scènes plus stylisées, oniriques, figurant des espaces de refuge fantasmés par Vincent. La violence se trouve déplacée : elle n'est plus le fait d'un acte meurtrier, mais se redéploie au contraire dans les pressions quotidiennes subies par le protagoniste. S'il n'est donc pas inscrit directement dans la trame narrative, le fait divers comme scénario dramatique demeure omniprésent, à la manière du miroir inversé. L'absence de toute velléité meurtrière ou suicidaire chez Vincent souligne précisément en quoi il n'est pas un personnage de fait divers, son caractère normatif ne lui permettant pas d'accéder à la subversion. La triste banalité du personnage est d'ailleurs soulignée par un choix de casting astucieux : Vincent, incarné par le comédien Aurélien Recoing, peu connu au cinéma, fait pâle figure devant l'acteur non-professionnel Serge Livrozet, ancien perceur de coffres, incarnant dans le film un trafiquant, dont la biographie est restituée dans un "pressbook» composé de coupures de presse relatant son procès. La confrontation avec ce personnage transmédiatique permet ainsi de souligner le manque de radicalité de Vincent: tenté un temps par la paradoxale sincérité qu'il trouve dans la marginalité, il se résout pourtant à quitter cet espace réconfortant dès lors qu'il met en danger sa relation avec sa femme Muriel.

\section{Le fait divers comme discours}

15 L'étude des phénomènes de circulation du fait divers dans les arts nécessite d'être contextualisée dans le double but de ne pas réifier cette matière proprement indisciplinée et d'éviter de reconduire au sein de l'analyse des grilles de lecture légitimistes. La mise à distance du registre de la sublimation implique cependant de reconsidérer l'attrait contemporain pour les faits divers. C'est pourquoi, sans vouloir retomber dans l'opposition largement dépassée entre fond et forme, nous aborderons désormais le fait divers comme discours ${ }^{16}$. Nous souhaitons ainsi faire apparaître le caractère socioculturel du genre, et inviter à réfléchir à la place jouée par sa dimension symbolique dans ses appropriations artistiques.

\section{Historiciser le discours fait-diversier : la monstration des rapports sociaux par l'intermédiaire d'individualités}

Si le fait divers renvoie le plus souvent à des mises en mots et en images spécifiques des désordres quotidiens, il constitue également un discours sociohistoriquement situé, dont les modalités sont étroitement liées aux transformations de l'écriture journalistique - une évidence importante à rappeler pour nuancer quelques-uns des présupposés servant de base à sa condamnation. Un certain nombre d'écrits contemporains, que Dominique Viart regroupe sous l'appellation de «fictions 
critiques ", tend ainsi à reprocher aux faits divers leur propension à véhiculer des visions fortement objectivées du monde. Contre cela, ces productions se constituent comme «mode d'investigation du réel»; «elles font du détour par la fiction une méthode pour déconstruire ces fictions du réel ou du verbe que proposent encore trop de discours ${ }^{17} »$. Ce refus de toute réalité réifiée traverse, dans des modalités non ouvertement critiques, les visions du monde proposées dans L'Adversaire et L'Emploi du temps : les deux longs-métrages ont en effet en commun d'être largement imprégnés de l'univers mental troublé de leur protagoniste. Ce phénomène se manifeste chez Garcia par le biais d'une narration profondément fragmentée, traduisant l'impossible restitution ordonnée du drame. Cet éclatement n'est pas sans refléter la confusion de Jean-Marc Faure, comme en témoignent les deux versions, présentes au début et à la fin $\mathrm{du}$ film, du retour du protagoniste dans le foyer familial une fois les meurtres commis : dans la première, Jean-Marc reproduit des rituels quotidiens - prendre le courrier, appeler sa femme - comme pour conjurer le drame, avant d'apercevoir sur le carrelage le bol de céréales de son fils; dans la seconde, le personnage, conscient de ses actes, lâche le courrier sur le sol et s'effondre dans un fauteuil. Le film de Laurent Cantet pose comme intermédiaire le rapport particulier de son protagoniste au monde extérieur, produisant ainsi une forte déréalisation de la vie quotidienne : Vincent tient en effet un rôle de spectateur du monde, et ne cesse de regarder les autres, leurs interactions, à travers des vitres. L'utilisation de la transparence, notamment architecturale, dans le film prend une dimension ironique dans la mesure où, loin de donner accès à l'essence des phénomènes, elle souligne leur formidable superficialité, leur dimension performative ${ }^{18}$.

17 Cette distanciation critique quant à la croyance en une réalité immanente n'est bien entendu pas sans consistance sociologique: elle fait écho à la progression de l'individualisme sous la seconde modernité, aux contradictions de plus en plus puissantes traversant la matière sociale et à la mise en question des institutions qu'elles supposent ${ }^{19}$. Si l'enjeu de crédibilité auquel doivent répondre les médias d'information les mène bien à souscrire à des "effets de vérité ", les médiations qu'ils effectuent du « réel» connaissent pourtant un bouleversement similaire. Reprenant la conceptualisation d'Antoine Hennion, Guy Lochard affirme ainsi que la télévision est passée du stade de la "représentation représentée ", donnant à voir "un monde qui existe en soi, indépendamment de nous " à celui de "représentations représentantes, c'est-à-dire un état où le monde ne prend sens et intérêt à nos yeux qu'en fonction de notre présence au monde ${ }^{20} »$. La promotion sans précédent d'anonymes au sein des reportages, la montée de paroles situées et individualisées que traduit l'avènement des figures de victime ou encore de d'avocat médiatique ${ }^{21}$, de même que le traitement de plus en plus prégnant des faits divers comme faits de société22 montrent en effet que le discours d'information tend également à donner accès aux rapports sociaux par l'intermédiaire d'individualités. Il semble partager avec les deux fictions cinématographiques, dans des modalités évidemment distinctes, la vision d'une " réalité située ", nécessairement médiée par des affects et des expériences singulières.

\section{Mettre en scène les angoisses identitaires contemporaines : l'errance derrière la violence rassurante des rôles} ainsi de relativiser certains des présupposés souvent avancés sur le genre, le 
rapprochant de ce fait des autres discours sociaux ; nous pensons qu'elle est également susceptible de mieux saisir son attrait contemporain : pourquoi, en effet, les faits divers font-ils l'objet de tant d'attention? Telle est la vaste question, éminemment complexe, à laquelle nous nous proposons de réfléchir en élaborant l'hypothèse suivante : les faits divers sont peut-être socioculturellement signifiants en ce qu'ils problématisent, dans leur mise en visibilité des crimes individuels, certaines des angoisses identitaires contemporaines. Ces récits meurtriers ont en effet la particularité de souvent présenter des citoyens ordinaires dont les crimes contredisent l'identité sociale. Cette mise en question radicale des «monsieur-et-madame-tout-le-monde " n'a rien d'atemporel mais provient de la mutation des figures criminelles entre le $\mathrm{xIX}^{\mathrm{e}}$ et le $\mathrm{xx}^{\mathrm{e}}$ siècles : autrefois signifiée par des difformités corporelles, la « monstruosité » des protagonistes s'est ainsi déplacée vers un imaginaire de la duplicité, susceptible de se loger chez tout un chacun $^{23}$. Le discours fait-diversier tendrait dès lors à mettre en évidence la disjonction contemporaine entre les rôles et les identités, ou plutôt les errances identitaires résultant de cette disjonction ${ }^{24}$.

Cette particularité du discours fait-diversier n'implique pas qu'il exerce sur les autres formes culturelles une autorité surplombante. Les angoisses identitaires, largement traitées dans le cinéma contemporain, sont loin d'être l'apanage des faits divers, mais renseignent peut-être sur ces phénomènes de circulation. Si L'Adversaire a fait l'objet de peu de commentaires, plusieurs travaux pointent d'ailleurs les façons dont L'Emploi $d u$ temps cherche à se saisir des crises rencontrées par les masculinités blanches contemporaines ${ }^{25}$. Ces problématiques sont en réalité présentes dans les deux fictions, qui mettent en scène les tentatives d'individus en mal d'eux-mêmes pour raccorder leur identité à un rôle, soulignant ainsi leur dimension proprement performative. Dans L'Adversaire comme dans L'Emploi du temps, la fonction du pourvoyeur économique, étroitement liée à l'identité de père de famille, est travaillée sans relâche par les protagonistes, à la manière d'un acteur : ils se rendent sur les lieux de leur emploi fictif pour en saisir l'atmosphère, ils étudient voire récitent les discours institutionnels, ils s'approprient les codes tant vestimentaires que comportementaux de leurs (faux) semblables. Leur performance est en définitive si bien jouée qu'elle leur permet de se fondre dans la masse, et d'avoir accès à l'univers de la famille relationnelle ${ }^{26}$. Habité par une fêlure identitaire forte, les deux personnages se montrent en définitive insatisfaits de leur propre platitude et se lancent dans l'aventure - relation extra-conjugale pour Jean-Marc Maure, trafic pour Vincent-comme pour pallier leur étouffement identitaire. Ces écarts précipiteront, dans les deux cas, la découverte du mensonge, soulignant ainsi la violence des rôles.

Objet culturel complexe, le fait divers souffre d'une forte dévalorisation. Vecteur privilégié des désordres ordinaires et des crimes sanglants, territoire d'excès et de sensations fortes, le fait divers ne peut pourtant être réduit à une information pauvre et voyeuriste. C'est pourquoi l'approche culturelle du genre est, à bien des niveaux, pertinente : elle permet de prendre acte des critiques qui lui sont adressées sans pour autant perdre de vue ses incarnations variées et facettes multiples. Ainsi, les jeux et contrastes stylistiques présents dans L'Emploi du temps et L'Adversaire ne peuvent être étudiés en réifiant cette matière première, mais dépendent des problématisations et rapports différenciés qu'entretiennent ces productions avec le fait divers. Penser le genre en termes de rapports de pouvoir permet également de l'appréhender comme discours. Il s'agit alors de considérer les manières, sociohistoriquement situées, dont il 
met en récit les désordres quotidiens. La mutation des figures meurtrières, propices à la monstration d'angoisses identitaires, explique peut-être une partie de son attrait contemporain. La dimension symbolique, profondément politique, des faits divers gagnerait ainsi à être davantage étudiée dans le but de mieux comprendre la complexité de ses circulations et de ses appropriations culturelles.

\section{NOTES}

1. Voir notamment : É. Brière, «Faits divers, faits littéraires. Le romancier contemporain devant les faits accomplis », Études littéraires, vol. 40, 2009, p. 157-171.

2. A. Dubied, M. Lits, Le Fait divers, Paris, Presses universitaires de France, 1999, p. 76.

3. M. Lits, « Le fait divers, un genre strictement francophone? ", Semen, $n^{\circ} 13,2001$, p. 37-47.

4. J. Mittell, « A Cultural Approach to Television Genre Theory », Cinema Journal, 40, 2001, p. 9.

5. Ibid., p. 8.

6. A. Dubied, Les Dits et les scènes du fait divers, Genève-Paris, Droz, 2004, p. 13.

7. La portée historique et sociologique du fait divers est néanmoins traitée par de nombreux travaux. Voir notamment : Cl. Sécail, Le Crime à l'écran : le fait divers criminel à la télévision française, 1950-2010, Paris, Nouveau monde, 2010 ; A.-C. Ambroise-Rendu, Petits récits des désordres ordinaires : les faits divers dans la presse française des débuts de la III République à la Grande Guerre, Paris, Seli Arslan DL, 2004.

8. A. Dubied, « S'inspirer du fait divers : comment et pourquoi ? » dans J. Migozzi et Ph. Le Guern (dir.), Production(s) du populaire, Limoges, Presses Universitaires de Limoges, 2004, p. 383.

9. R. Barthes, "Structure du fait divers ", Essais Critiques, Paris, Seuil, 2000, p. 194-204.

10. Ces critiques sont également fréquentes dans les discours portés par les artistes. Voir notamment : É. Brière, « Faits divers, faits littéraires ", art. cit., p. 165.

11. D. Viart, "Fiction et faits divers", La Littérature française au présent. Héritage, modernité, mutations, Paris, Bordas, coll. « La Bibliothèque Bordas », 2008, p. 235-251.

12. Studio Magazine, mai 2002, p. 146. Cité dans A. Dubied, « S'inspirer du fait divers : comment et pourquoi ?», art. cité.

13. J. Kermabon, « Entretien avec Laurent Cantet », 24 Images, $n^{\circ} 100$, printemps 2002, p. 32.

14. Y. Lebtahi, I. Roussel-Gillet, Pour une méthode d'investigation du cinéma de Laurent Cantet: les déplacés, vertiges de soi, Paris, L'Harmattan, 2005, p. 37.

15. Entretien de Nicole Garcia publié sur le site Ecran Noir: <www.ecrannoir.fr/entrevues/ entrevue.php ?e =174\&p =2> (consulté 14/05/2017).

16. M. Foucault, L'Ordre du discours, Paris, Gallimard, 1971.

17. D. Viart. "Les "fictions critiques" de la littérature contemporaine ", Spirale, $\mathrm{n}^{\circ}$ 201, 2005, p. 10-11.

18. La performativité est ici entendue dans le sens que lui confère Judith Butler, soit comme renvoyant "au caractère "dramatique" et contingent de la construction de la signification ». J. Butler, Trouble dans le genre, C. Kraus (trad.), Paris, La Découverte, 2006, p. 263.

19. Voir notamment: D. Martuccelli et Fr. de Singly, Les Sociologies de l'individu, Paris, Armand Colin, 2012.

20. G. Lochard, «La parole du téléspectateur dans le reportage télévisuel », dans J.-P. Esquénazi (dir.), La Télévision et ses spectateurs, Paris, L'Harmattan, 1995, p. 151. 
21. Cl. Sécail, Le Crime à l'écran, ouvr. cité.

22. M. M'Sili, « Du fait divers au fait de société ( $\mathrm{XIX}^{\mathrm{e}}-\mathrm{XX}{ }^{\mathrm{e}}$ siècles) : les changements de signification de la chronique des faits divers ", Les Cahiers du journalisme, $\mathrm{n}^{\circ} 14,2005$, p. $30-45$.

23. S. Châles-Courtine, «La construction de figures criminelles dans les faits divers du $\mathrm{XIX}^{\mathrm{e}}$ et $\mathrm{Xx}^{\mathrm{e}}$ siècles », dans C. Dessinges et I. Garcin-Marrou (dir.), Média et Culture, numéro spécial, mars 2008, p. 57.

24. J.-C. Kaufmann, L'Invention de soi : une théorie de l'identité, Paris, Armand Colin/SEJER, 2004.

25. Voir notamment : N. Archer, « The Road as the (Non)-Place of Masculinity : L' Emploi du temps ", Studies in French Cinema, vol. 8, n² 2, 2008, p. 137-148; J. Franco, "'The More You Look, the Less You Really Know' : The Redemption of White Masculinity in Contemporary American and French Cinema ", Cinema Journal, vol. 47, nº 3, 2008, p. 29-47; W. Higbee, "'Elle est où, ta place ?' The Social-Realist Melodramas of Laurent Cantet», French Cultural Studies, vol. 15, $\mathrm{n}^{\circ}$ 3, 2016, p. 235-250.

26. Fr. de Singly, Sociologie de la famille contemporaine, Paris, Armand Colin, 2014.

\section{RÉSUMÉS}

Les représentations des faits divers sont loin d'être indemnes d'ambiguïtés: loués pour les cristallisations qu'ils opèrent de problématiques tant humaines que sociales, les faits divers demeurent dévalués dans leur matérialité journalistique. Ce paradoxe mène à aborder la notion de genre comme catégorie culturelle (Mittell, 2001), soit comme pratique discursive située à l'intersection de rapports de pouvoir. Cette perspective est illustrée par l'analyse de deux fictions inspirées de l'affaire Romand, L'Adversaire de Nicole Garcia et L'Emploi du temps de Laurent Cantet. Elle permet d'envisager les appropriations et jeux stylistiques indépendamment du registre de la sublimation, fréquemment mobilisé pour caractériser le passage des médias aux arts. Elle est également un outil intéressant pour considérer l'attrait des faits divers du point de vue de leur dimension symbolique, et des façons dont ils traduisent certaines des angoisses identitaires contemporaines.

The representations of the faits divers are ambiguous: prized for their reflection of complex human and social issues, they are at the same time devalued in their journalistic dimension. This paradox leads to one considering the genre as a cultural category (Mittell, 2001), and as a discursive practice situated at the intersection of many power relations. This perspective is illustrated through the analysis of two works of fiction inspired by the Romand case: Time Out (Laurent Cantet) and The Adversary (Nicole Garcia). This point of view helps to understand the circulation and the stylistic appropriations outside the scope of sublimation, often used to qualify the transition from media to arts. It is also an interesting tool to approach the attractiveness of the faits divers through their symbolic dimension and the ways in which they convey contemporary identity anxieties. 


\section{AUTEUR}

\section{SOPHIE DUBEC}

Université Sorbonne Nouvelle (Paris 3), IRMECCEN (EA 1484)

Sophie Dubec est doctorante en sciences de l'information et de la communication à la Sorbonne Nouvelle, Paris 3, et associée au laboratoire CIM-IRMECCEN. Sa thèse, située à la croisée de la sociologie des médias et de l'individualisme, ainsi que des Gender Studies, porte sur les reconfigurations des maternités et paternités, des masculinités et féminités, que cristallisent les mises en récit des parents infanticides dans les journaux télévisés français. 